\title{
Using hardware interrupts for timing visual displays and reaction-time key interfacing on the Commodore 64
}

\author{
RICHARD D. WRIGHT \\ The University of Western Ontario, London, Ontario, Canada \\ and \\ MICHAEL R. W. DAWSON \\ York University, Toronto, Ontario, Canada
}

\begin{abstract}
The use of hardware interrupts for presenting and timing visual displays and for controlling reaction timing on the Commodore 64 is described. The three sources of interrupts discussed are reaction-time keys interfaced through the user port to on-board hardware timers, the alarm of the $60-\mathrm{Hz}$ real-time clocks, and the video raster. In a demonstration program, these interrupts are used to measure display durations, to change screen displays, and to coordinate the onset of reaction timing with the onset of screen changes. In addition, an externally generated interrupt caused by a keypress is used to control reaction timing independently of CPU operations.
\end{abstract}

The Commodore 64 is an inexpensive machine, yet it has many features that make it an attractive choice as a laboratory computer (see Kallman, 1986). One of these is that the hardware interrupts can be programmed quite easily for precise control over the presentation and timing of visual displays. This is not the case with some other microcomputers. For example, the Apple II requires a clockcard and other hardware modifications to the motherboard for the same type of flexibility (see Reed, 1979).

This paper provides a discussion of the use of Commodore 64 interrupts for synchronizing and timing visual displays and for controlling the on-board hardware timers. Also, an example program is described for using the Commodore 64 as a two-field tachistoscope. When the program is running, a field containing a fixation cross (Field 1) is displayed for a specified duration, and then an interrupt signal is generated. This signal causes Field 2 to replace Field 1, starts a millisecond reaction timer, and turns on a hardware alarm. The subject's task is to search Field 2 for the presence or absence of a particular target object. Pressing one of the two reaction-time keys at this

The writing of this report was supported by a Natural Sciences and Engineering Research Council of Canada (NSERC) postgraduate scholarship awarded to the first author, a York University President's NSERC grant awarded to the second author, and NSERC Grant A2600 awarded to Zenon Pylyshyn. We are grateful to Jim Clark for directing us to documentation on the Commodore 64 , as well as for making helpful comments on this manuscript. We also thank Barbara Snyder for her comments on the manuscript and Dan Pulham for his assistance in the design and construction of the reaction-time key mechanism. Michael Dawson is now at the Centre for Advanced Study of Theoretical Psychology at the University of Alberta. Requests for reprints should be sent to Richard D. Wright, Centre for Cognitive Science, Department of Psychology, University of Western Ontario, London, ON N6A 5C2, Canada. point produces an interrupt that causes the response and the latency to be recorded. In addition, a keypress causes Field 2 to be replaced by a text screen. If a key is not pressed before a particular time interval has elapsed $(3 \mathrm{sec})$, the time-of-day clock sends an interrupt signal that ends the trial and turns off Field 2. Different parts of this demonstration program are referred to in the following discussion.

\section{Programming Interrupt Routines}

Interrupts on the Commodore 64 interrupt the program currently being carried out by the $6510 \mathrm{CPU}$ and cause it to execute an interrupt routine before returning to the original program. Interrupts are signals generated by hardware devices and can occur at any time within a program. One example is the system interrupt. Every $16.67 \mathrm{msec}$, one of the 6526 complex interface adapters (CIAs) generates an interrupt signal that stops execution of the current program and starts the system "housekeeping" routine. Among the operations carried out at this point are a cursor flash, a scan of the keyboard buffer, and an increment of the jiffy clock counter. Execution of the interrupted program then resumes until the next interrupt occurs. Because the routines that are run when an interrupt signal is detected typically takes less than a millisecond to be carried out, they are essentially "invisible" to other operations of the CPU (e.g., the running of a BASIC program).

Two types of interrupt signals can be generated within the Commodore 64. One is a nonmaskable interrupt (NMI) signal. This type of interrupt can be generated by the RESTORE key, the CIA \#2 timers and time-of-day clock, and an external device interfaced to the FLAG pin of the user port. The second type of interrupt is an inter- 
rupt request (IRQ) signal. This signal can be generated by the CIA \#1 timers and time-of-day clock, and by the raster beam register, which keeps track of the location of the raster beam on the video screen. The difference between an NMI signal and an IRQ signal is that the latter can be ignored by setting the interrupt flag of the CPU, whereas an NMI signal cannot be ignored.

When an interrupt signal is sent, the following occurs: First, the current values of the program counter and the status flags are saved on the system stack. These values are recalled at the end of the interrupt routine so that the interrupted program can run normally. Second, control of the CPU is passed to a specific program (the interrupt routine). This is accomplished by examining the contents of two RAM addresses that contain the least significant and most significant bytes of the starting address of the interrupt routine. These addresses are referred to as an interrupt vector. For example, when an IRQ signal is detected, program control is passed to the routine whose starting location is contained at addresses \$0314 and \$0315 (note that the $\$$ prefix denotes hexadecimal numbers). Usually the addresses contain the values $\$ 31$ and \$EA such that the IRQ interrupt vector points to the system interrupt housekeeping routine starting at \$EA31. When an NMI signal is detected, program control is passed to the NMI interrupt vector at addresses \$0318 and \$0319, which usually points to the interrupt routine starting at \$FE47.

Two steps are involved when programming interrupts. First, a machine code interrupt routine must be written that performs the operations that are to occur as a result of interrupt signals. Second, the contents of the RAM locations examined when interrupts occur must be changed to point to the new interrupt routine instead of to the default routine. The INITIALIZE routine listed in the Appendix illustrates these steps. Part of this routine involves changing the contents of locations $\$ 0314$ and $\$ 0315$ to point to the IRQTEST subroutine instead of to the normal IRQ server. After this change has been made, an IRQ signal causes IRQTEST to run. Similarly, the contents of locations $\$ 0318$ and $\$ 0319$ are changed to point to the NMITEST subroutine instead of to the normal NMI server.

It should be pointed out that when an IRQ interrupt signal is detected, the values of the accumulator, $X$, and $Y$ registers of the CPU at the time of the interrupt are saved on the system stack before program control is vectored through $\$ 314$ and $\$ 315$. The register values must be restored before control is returned to the foreground program, or this program will crash. One way to do so is to exit from an IRQ interrupt routine through the system interrupt routine. The system interrupt then pulls these values off the stack and loads them into the appropriate registers before returning to the foreground. This is the approach we have used in the IRQTEST subroutine in the Appendix (see line \$C086). Those researchers who do not want to exit from an IRQ routine through the system interrupt, perhaps to avoid the extra time required for housekeeping, must restore the register values before a direct return to the background. This is done by pulling the $Y$ register value off the stack with the PLA command and transferring it to this register with the TAY command. The $\mathrm{X}$ register value is then pulled off the stack with the PLA command and transferred to this register with the TAX command. Finally, the accumulator value is pulled off the stack with the PLA command, and a return to the foreground program is made with the RTI command. A similar procedure is used in the NMITEST subroutine in the Appendix for restoring the $\mathrm{Y}$ register and accumulator values (see lines $\$ C 103$ to $\$ C 106$ ). In the sections that follow, we describe how IRQTEST and NMITEST are used to control the timing of displays and the measurement of response latencies.

\section{A Millisecond Reaction Timer}

The Commodore 64 uses two CIA chips to control its input/output operations, and both chips are available for programming by the user. Each CIA chip contains two 16-bit, 1.022-MHz timers that can be cascaded for timing short durations with millisecond accuracy or greater, and a $60-\mathrm{Hz}$ time-of-day clock for timing relatively long durations, but with less precision. Both types of clocks run independently of the CPU. We have found this to be very useful for measuring reaction times in tasks such as the visual tracking of moving targets presented on a cathode ray tube (Pylyshyn, 1988) because responses can be timed independently of the CPU operations that move the targets.

Multiple sources of hardware timing allow for the precise control over durations of different aspects of an experiment. For instance, in the programs described in this paper, the 16-bit clocks are used to measure reaction times, while the time-of-day clock is used to limit the viewing time of Field 2 to $3 \mathrm{sec}$.

In the remainder of this section, we describe how to set up a millisecond timer that is completely independent of the Commodore 64 CPU. In particular, the programming of the 16-bit clocks of CIA \#2 for millisecond timing and the construction of a response key mechanism that produces NMI interrupt signals when its keys are pressed are described. Also, an NMI interrupt routine is given for interfacing these two devices so that a keypress stops the millisecond timer and causes data to be recorded.

The two 16-bit timers (Timer A \& Timer B) of CIA $\$ 2$ are available to the user except when required by the operating system for serial communications. These timers can be cascaded so that Timer B decrements a counter every time Timer A counts down to zero (i.e., when Timer A underflows). Thus if Timer A is programmed to underflow every millisecond, Timer B records elapsed time in milliseconds. Six registers must be set to enable this type of timing. This is illustrated in the TIMER SETUP subroutine listed in the Appendix. Control Register B (located at \$DDOF) of CIA $\$ 2$ must have bits 0 and 6 set to 1 . This causes Timer B to count down Timer A underflow pulses. Timer A is programmed to underflow ev- 
ery millisecond by setting its low byte register (\$DD04) to $\$ F C$ and its high byte register (\$DD05) to \$03. Timer B is initialized as a counter by setting its low byte (\$DD06) and high byte (\$DD07) registers to \$FF. The clocks are now programmed to operate as a millisecond timer. The timer is started by setting bit 0 of Control Register A (\$DD0E) to 1 and stopped by setting this bit to 0 . This occurs in the demonstration program when a reaction-time keypress produces an NMI signal (see NMITEST in the Appendix). The Timer B low and high bytes can then be examined to read the reaction-time (see line 400 in the demonstration program shown in Listing 1). A similar procedure for programming the CIA $8520 \mathrm{~A}$ chips of the Commodore Amiga for cascaded timing has been described by Wright (1986).

For hardware timing to be completely independent of CPU operations, it is not sufficient to have an independently running hardware timer. The timer must be stopped by an externally generated signal that is independent of the CPU. This can be accomplished by interfacing reaction-time keys to the CIA \#2 FLAG line through the user port. A signal over this line will produce an NMI signal that can be used to call an interrupt routine that stops the timer. With this external control over interrupts, there is no need for the CPU to repeatedly poll a memory location waiting to detect a keypress. Instead, other operations can be carried out by the CPU during the timed interval.

Often, timing algorithms do not have this independence from the CPU. For example, in a program described by Horrman and Allen (1987), the CPU is used to repeatedly poll the raster compare register during the screen switch routine for a match between the value stored in this register and the video line that the raster beam is currently scanning. During the timing routine, the $\mathrm{CPU}$ is used to repeatedly poll a memory location for a keypress. Thus the majority of CPU time is taken up by polling operations. However, if hardware interrupts are used, timing and screen switching can be carried out with a minimum of CPU time. Hence the CPU is free for such operations as producing dynamic visual displays during the timed intervals.

To enable the FLAG line of the user port to generate an NMI signal, bits 4 and 7 of the CIA \#2 interrupt control register (\$DD0D) must be set to 1. Note that an NMI interrupt is more desirable than is an IRQ for stopping reaction-time clocks. Unlike IRQ signals, an NMI signal cannot be temporarily disabled while other interrupt routines are being carried out. Hence there is no danger of the reaction times being erroneously inflated.

Figure 1 is a schematic wiring diagram of two reactiontime keys interfaced to the user port FLAG line. Each key is a single pole button with one line wired to ground (pin A) and the other line wired through a flip-flop switch to a data line and through a one-shot to the FLAG line. When a keypress occurs, the one-shot produces a $10-\mathrm{msec}$ "go-low" pulse (a 10-msec change from $+5 \mathrm{~V}$ to $0 \mathrm{~V}$ ) that is sent to the FLAG line (pin B). In addition, the flip-

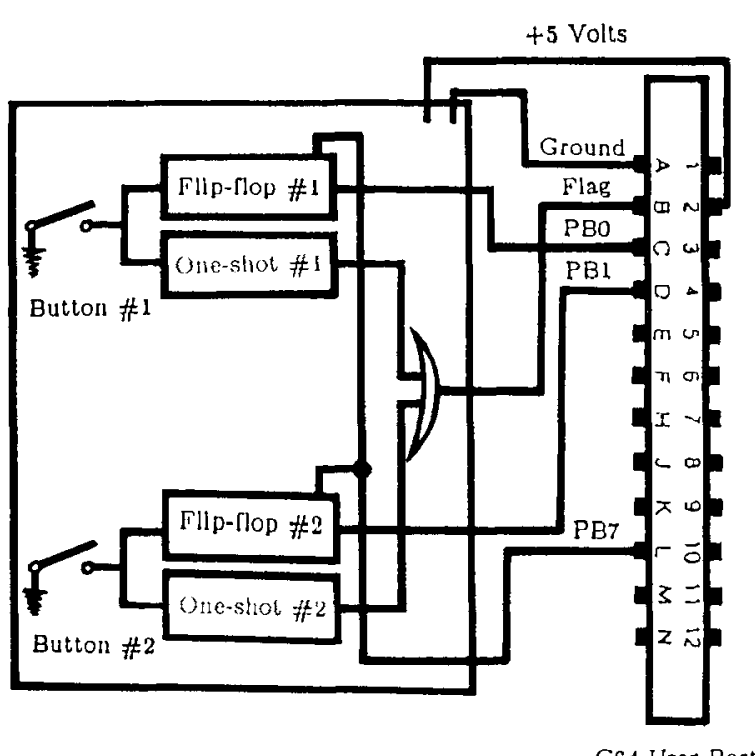

C64 User Port

Figure 1. A schematic wiring diagram of the reaction-time keys interfaced to the Commodore 64 user port.

flop switch keeps the data line wired to that key low $(0 \mathrm{~V})$. The purpose of the flip-flops is to hold the data lines low after the keypress has occurred so that an interrupt routine can read the data register (\$DD01) to determine which of the two keys was pressed. Keypresses can be differentiated by the associated data line (PBO or PB1) that was also driven low. Once the register has been read, a 1-msec pulse from the data line PB7 (pin L) is sent by the interrupt routine to reset the flip-flops so that data lines PB0 and $\mathrm{PB} 1$ are high again $(+5 \mathrm{~V})$.

The routine NMITEST listed in the Appendix enables external control of the millisecond timer by response keypresses. In particular, when an NMI signal occurs after INITIALIZE has been run, NMITEST performs the following operations: First, the millisecond timer is stopped by turning bit 0 of CIA \#2 Control Register A off. Following this, the counter bytes of Timer B and the contents of the user port register (\$DD01) are saved in RAM so that BASIC can determine which key was pressed and what the response latency was. Then a $1-\mathrm{msec}$ pulse or $0 \mathrm{~V}$ is sent through the user port (line PB7 in Figure 1) to reset the flip-flops in the response key mechanism. Finally, Field 2 is replaced by a text screen, and program control returns from the interrupt.

\section{Interrupts from Time-of-Day Clocks}

At the start of the previous section, we noted that in addition to the 16-bit timers, the CIA chips also have a time-of-day (TOD) clock. This clock keeps time with tenths of a second resolution and can be programmed to produce interrupts that are independent of those produced by the 16-bit timers. Thus, on the Commodore 64 there are multiple sources of interrupts that allow for precise control over the durations of different aspects of an experiment. In this section, programming the CIA \#2 TOD 
clock to generate NMI interrupts is described. In the demonstration program, this interrupt limits the amount of time that a display can be viewed. Note that the CIA \#1 TOD clock can also be programmed in this way, but it produces an IRQ rather than an NMI signal.

The TOD clock uses four registers to keep time, one each for tenths of seconds (located at address \$DD08), seconds (\$DD09), minutes (\$DD0A), and hours (\$DD0B). By setting bit 7 of CIA \#2 Control Register B (\$DD0F) to 0, writing to these four registers sets the clock's time. By setting bit 7 to 1 , writing to these registers sets the alarm time. To enable the TOD to generate an NMI interrupt, bits 2 and 7 of the CIA \#2 interrupt control register (\$DD0D) must be set to 1 . When the alarm is reached, an NMI signal is generated. The TOD clock is stopped by a read or write to the hours register, and is started by a read or write to the tenths of a second register. In the Appendix the machine language routine ALARM illustrates the programming of this clock. In this routine, the clock is set to 0 and the alarm time is set for $3 \mathrm{sec}$. Note that the TOD clock starts to run as soon as the tenths of a second register is set.

The four registers of the TOD clock represent time in binary coded decimal (BCD) format. In BCD format, a byte is separated into two 4-bit nibbles. Each nibble represents one digit of a base 10 number. For example, in the ALARM routine the alarm time seconds register could be set to $\$ 10$, which is BCD format for 10 , rather than $\$ 0 A$ (the hexadecimal equivalent to the decimal value of 10). The latter is not a proper BCD code for any value because " $A$ " is not a base 10 digit. If $B C D$ format is not used to program this clock, it is possible to set the alarm for a time that will never be reached because it is not a $B C D$ value (e.g., \$AC).

When an NMI signal is produced by the TOD alarm, the same operations as those caused by a keypress will be carried out in the demonstration program (i.e., the NMITEST routine). This is because the NMI RAM vector points to this routine regardless of the source of the NMI signal. For the type of task carried out by the demonstration program, this is not a problem. In particular, when an NMI signal occurs, the response latency and the value of the user port register are recorded regardless of whether the source of the interrupt signal was a keypress or the TOD alarm. If one wishes to have one set of operations result from a keypress and another set result from the alarm, this can be accomplished by having the NMITEST routine examine the CIA \#2 interrupt control register (\$DD0D). If bit 2 has been set to 1 , then the interrupt signal was produced by the TOD alarm. If bit 4 has been set to 1 , then the interrupt signal was produced by the FLAG line (a keypress), and a different set of operations should be invoked.

\section{VIC-II Raster Interrupts}

Cathode ray tubes (CRTs) using the American Standard Display are made up of 262 horizontal lines, 200 of which are visible (video lines 50-249). Each line is scanned 60 times per second, and, as a result, the exposure duration of stimuli presented on a CRT is $16.67 \mathrm{msec}$ and increments of this temporal interval. If a sequence of screens is to be displayed, care must be taken to switch from one to another while the nonvisible portion of the screen is being scanned. Otherwise, "screen shred" may occur, such that for a brief period of time the bottom of the screen shows the new stimulus while the top still shows the old stimulus.

The characteristics of the CRT display are controlled by the VIC-II graphics chip. One of the properties of this chip is that it stores the position of the raster beam as it sweeps across the CRT. This information can be used to generate an IRQ signal when a desired line is swept by the raster. In this way, it is possible to wait until the scan has reached a desired video line before changing the graphics display.

In addition to preventing the disruption of screen graphics, the raster interrupt can play an important role in the timing of visual displays when millisecond accuracy is required. Reaction timers should be started as stimulus onset occurs. Hence a machine language subroutine is required to start the timer at the same video line on each trial. If this is not done, there may be up to $16.67 \mathrm{msec}$ of variability in the reaction time that could obscure small but significant effects (see Lincoln \& Lane, 1980). Using a PET/CBM computer, Merikle, Cheesman, and Bray (1982) synchronized display and timing onsets with the rollover of the jiffy clock. The Commodore 64 is more flexible than this because timing and display onsets can be synchronized by the VIC-II raster interrupt with the scan of any video line on the CRT.

In the demonstration program, the VIC-II chip is set to generate an IRQ interrupt signal when video line 250 (not part of the visible screen) is reached by the raster beam. In addition, the raster interrupt is used to time the duration of a display by incrementing a counter of the number of sweeps of line 250 (i.e., 16.67-msec intervals) that have elapsed since the display was turned on. This interrupt is also used to start the millisecond timer and to switch the first display to a second display when a desired time interval has elapsed (see IRQTEST in the Appendix).

To program a raster interrupt, the raster compare register (\$D012) must be set to the video line at which the interrupt is to occur. Bit 7 of \$D011 must also be set because it is the "ninth bit" of the value stored in the raster compare register. This extra bit is required because there are 262 video display lines, but only 255 can be represented as an 8-bit value. The INITIALIZE routine demonstrates how to code video line 250 in this manner. When this has been done, bits 0 and 7 of the video interrupt mask register (\$D01A) must be set to 1 to enable the interrupt.

In addition to turning on Field 1, the START TRIAL routine enables the raster interrupt and uses it to precisely time the duration of the Field 1 display in 16.67-msec intervals and to initiate the switch between Field 1 and 
Field 2 while the raster beam is not on a visible line (to prevent screen shred). The INITIALIZE routine sets a RAM counter to a value of 90 , which the IRQTEST routine decrements each time the VIC-II generates an IRQ signal. When the counter reaches zero (i.e., after 90 raster sweeps of the display screen), the IRQTEST routine disables the raster interrupts by resetting the video interrupt mask register (\$D01A) and turns on the Field 2 display.

To change the CRT display from one field to another, the upper 4 bits (the upper nybble) of the memory control register (\$D018) must be changed. This is done by loading the accumulator with the value of this register (i.e., LDA \$D018) and setting the upper nybble of this value to zero with the command AND \#\$F (see, e.g., lines $\$ C 07 \mathrm{C}$ to $\$ \mathrm{C083}$ of the IRQTEST subroutine). The upper nybble is then set with the OR command depending on the particular portion of video memory that is to be displayed. For example, setting all 4 bits to one (i.e., OR $\# \$ F 0$ ) will cause video memory from $\$ 3 C 00$ to $\$ 4000$ to be displayed as soon as the result is stored in the memory control register (i.e., STA \$D018). This technique is valuable for carrying out the rapid switching necessary for animation and other types of dynamic visual displays.

\section{A Visual Search Program Incorporating Hardware Interrupts}

In a visual search experiment (e.g., Treisman, 1986), subjects are typically presented with an array of objects and are asked to detect a particular target within this array. The BASIC program in Listing 1 carries out a simplified visual search experiment to provide an example of how the three interrupt sources discussed above can be used. In this task a fixation cross is presented for a brief period of time $(1,500 \mathrm{msec})$, followed by the presentation of the stimulus display and the initiation of reaction timing. Subjects search the stimulus display for the presence of a target that differs from the distractors and press a response key to stop the timer. The machine language subroutines in the Appendix carry out these oper-

Listing 1

A BASIC Program for Loading and Calling the Machine Language Routines in the Appendix, and for Carrying Out a Visual Search Demonstration Experiment

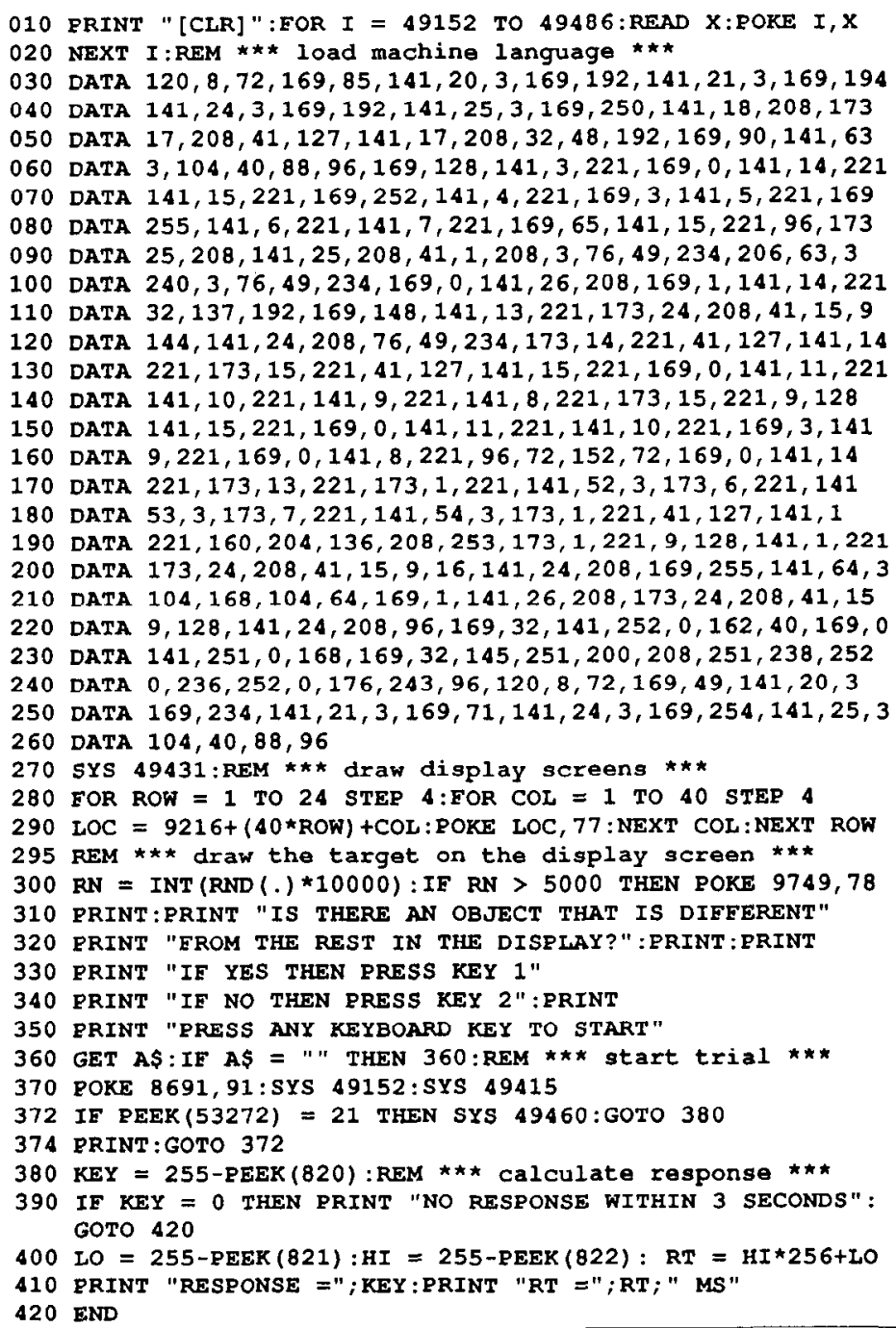


ations. For those without access to an assembler, the BASIC program in Listing 1 stores these routines in memory with POKE statements.

The program in Listing 1 works in the following way: First, the machine language subroutines are poked into memory at the start of the program and the SCREEN BLANK subroutine (see Appendix) is called by the command SYS 49431 to clear the display screens. Following this, a fixation cross is poked into the memory locations that contain the Field 1 display, and an array of objects is poked into Field 2. The interrupt routines are initialized by the command SYS 49152, and the trial is started by the command SYS 49415 . At this point, the interrupt routines switch the display from Field 1 to Field 2 after $1,500 \mathrm{msec}$ ( 90 raster sweeps of the screen) and start the reaction timer and alarm as described above. The interrupt vectors are reset by the command SYS 49460 (see the INTERRUPT RESET routine in the Appendix). Then BASIC examines the memory locations of the user port and the Timer B low and high bytes to calculate the reaction time for a response and to determine which key was pressed (if any).

If more than two aspects of an experiment are to be timed, the CIA \#1 chip can also be used to generate IRQs. Its TOD clock is always available, but the 16-bit timers are used to invoke the system interrupt routine. If this routine is disabled, the keyboard buffer is no longer scanned. Therefore, the CIA \#1 16-bit timers should only be used if keyboard input is not required during the experiment.
External control over these timers can be enabled by interfacing a response mechanism to the FLAG line of the cassette port. By taking advantage of the hardware timing and interrupt capabilities of the CIA and VIC-II chips, the programmer has a great deal of flexibility in designing experiments because the CPU is free to carry out other operations in addition to polling memory locations.

\section{REFERENCES}

Horrman, C. A., \& Allen, J. D. (1987). An accurate millisecond timer for the Commodore 64 or 128. Behavior Research Methods, Instruments, \& Computers, 19, 36-41.

Kallman, H. J. (1986). A Commodore 64-based experimental psychology laboratory. Behavior Research Methods, Instruments, \& Computers, 18, 222-227.

LINCOLN, L. E., \& LANE, D. M. (1980). Reaction measurement errors resulting from the use of CRT displays. Behavior Research Methods \& Instrumentation, 12, 55-57.

Merikle, P. M., Cheesman, J., \& Bray, J. (1982). PET Flasher: A machine language subroutine for timing visual displays and response latencies. Behavior Research Methods \& Instrumentation, 14, 26-28. Pylyshyn, Z. W. (1988). Here and there in the visual field. In Z. W. Pylyshyn (Ed.), Computational Processes in Human Vision: An Interdisciplinary Approach. Norwood, NJ: Ablex.

Reed, A. V. (1979). Microcomputer display timing: Problems and solutions. Behavior Research Methods \& Instrumentation, 11, 572-576.

Treisman, A. M. (1986). Features and objects in visual processing. Scientific American, 255(5), 114-125.

Wright, R. D. (1986). Amiga 1000 hardware timing and reaction-time key interfacing. Behavior Research Methods, Instruments, \& Computers, 18, 463-465.

\section{APPENDIX}

\begin{tabular}{|c|c|c|c|}
\hline \multirow{3}{*}{$\begin{array}{l}C 000 \\
C 001\end{array}$} & & \multirow[t]{2}{*}{ INITIAUIZE } \\
\hline & SEI & & \\
\hline & PHP & & \\
\hline $\mathrm{COO} 2$ & PHA & & \\
\hline $\mathrm{co03}$ & LDA & $\# \$ 55$ & ; Change IRQ RAM vector to \$C055 \\
\hline $\operatorname{co0} 5$ & STA & $\$ 314$ & \\
\hline $\mathrm{C008}$ & LDA & $\# \$ C 0$ & \\
\hline COOA & STA & $\$ 315$ & \\
\hline COOD & LDA & $\# \$ C 2$ & ; Change NMI RAM vector to $\$ C O C 2$ \\
\hline COOE & STA & $\$ 318$ & \\
\hline $\mathrm{C} 012$ & LDA & $\# \$ C O$ & \\
\hline $\mathrm{C} 014$ & STA & $\$ 319$ & \\
\hline $\mathrm{C} 017$ & LDA & \#\$FA & ; Set Raster Compare Register to 250 \\
\hline $\mathrm{C} 019$ & STA & $\$ D 012$ & \\
\hline $\mathrm{CO}$ & LDA & \$D011 & ; Set "ninth bit" of Raster Compare \\
\hline $\mathrm{C} 01 \mathrm{~F}$ & AND & $\# \$ 7 F$ & Register \\
\hline $\mathrm{C} 021$ & STA & $\$ D 011$ & \\
\hline $\mathrm{C} 024$ & JSR & $\$ C 030$ & ; Jump to TIMER SETUP subroutine \\
\hline $\mathrm{C} 027$ & LDA & $\# \$ 5 A$ & ; Set a counter to $90--$ this counter \\
\hline $\operatorname{co2} 9$ & STA & $\$ 335$ & is decremented by IRQTEST \\
\hline $\mathrm{Co2C}$ & PIA & & \\
\hline $\mathrm{CO} 2 \mathrm{D}$ & PLP & & \\
\hline $\operatorname{Co2E}$ & CLI & & \\
\hline $\mathrm{CO} 2 \mathrm{~F}$ & RTS & & \\
\hline & & & TIMER SETUP \\
\hline $\mathrm{C} 030$ & IDA & $\# \$ 80$ & ; Set data direction register \\
\hline $\cos 2$ & STA & \$DD03 & \\
\hline $\mathrm{C035}$ & LDA & $\# \$ 0$ & ; Set CIA \#2 registers for cascaded \\
\hline $\mathrm{CO} 37$ & STA & \$DDOE & timing \\
\hline C03A & STA & \$DDOF & \\
\hline C03D & LDA & $\# \$ F C$ & ; Set Timer A low byte \\
\hline C03E & STA & \$DD04 & \\
\hline
\end{tabular}




\section{APPENDIX (Continued)}

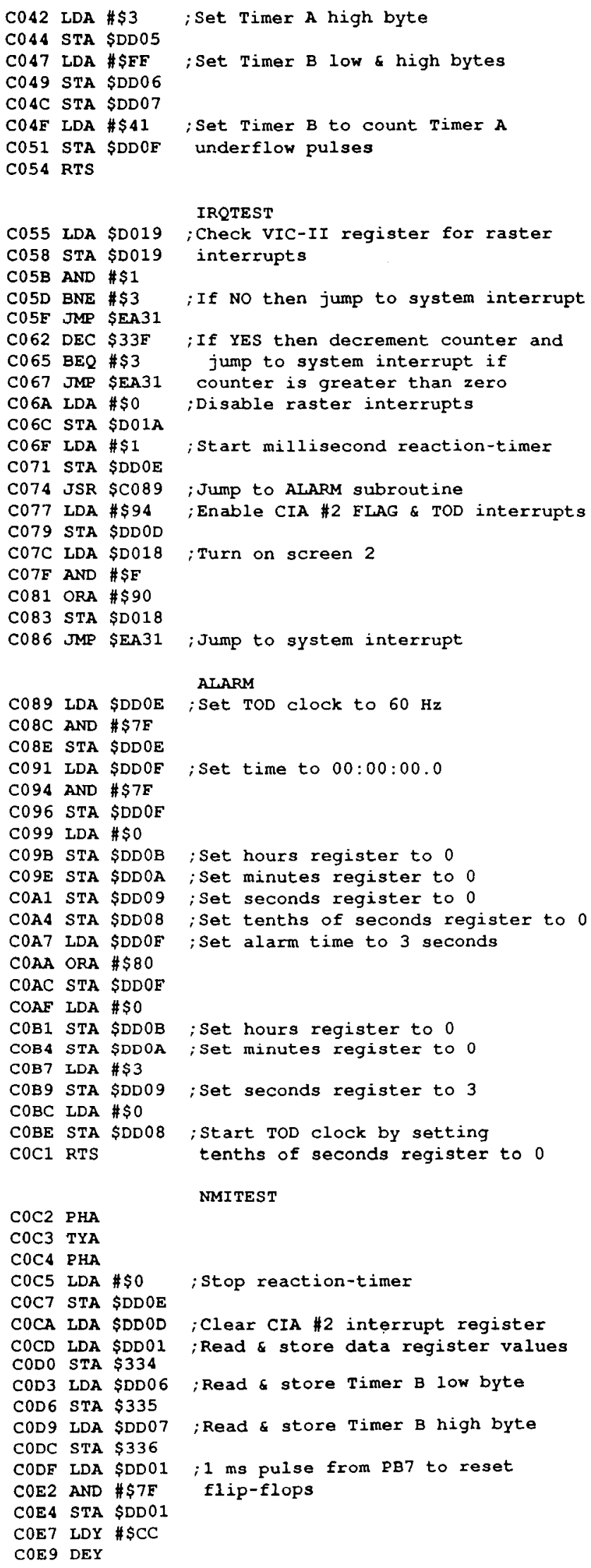


APPENDIX (Continued)

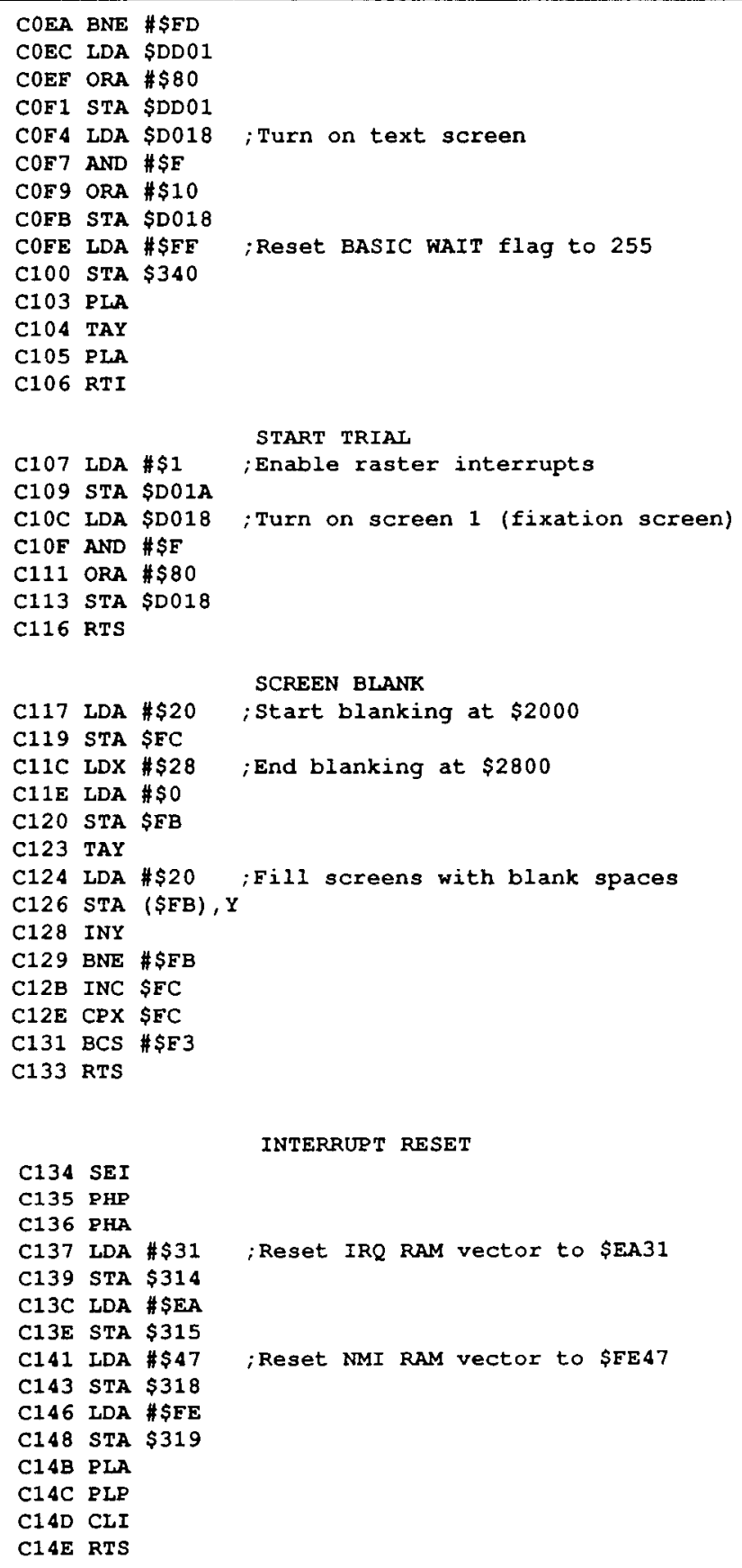

C137 LDA \#\$31 ; Reset IRQ RAM vector to \$EA31

INTERRUPT RESET

; Reset NMI RAM vector to \$FE47

(Manuscript received June 15, 1987; revision accepted for publication October 22, 1987.) 